Maltepe Journal of Mathematics

ISSN:2667-7660, URL:HTTP://DERGIPARK.ORG.TR/TR/PUB/MJM

Volume III Issue 1 (2021), Pages 30-51. Doi:Https://DOI.org/10.47087/MJM.911431

\title{
STABILITY ANALYSIS OF A NOVEL ODE MODEL FOR HIV INFECTION
}

\author{
HOANG ANH NGO*, HUNG DANG NGUYEN**, AND MEHMET DIK*** \\ *ÉCOLE POLYTECHNIQUE, INSTITUT POLYTECHNIQUE DE PARIS, 91120 PALAISEAU, \\ FRANCE \\ **VIETNAMESE - GERMAN FACULTY OF MEDICINE (VFGM), PHAM NGOC THACH \\ UNIVERSITY OF MEDICINE, HO CHI MINH CITY 710000, VIETNAM \\ ***DEPARTMENT OF MATHEMATICS AND COMPUTER SCIENCE, BELOIT COLLEGE, \\ WI 53511, UNITED STATES
}

\begin{abstract}
In this paper, we propose and investigate the stability of a novel 3-compartment ordinary differential equation (ODE) model of HIV infection of $\mathrm{CD} 4^{+}$T-cells with a mass action term. Similar to various endemic models, the dynamics within the model is fully determined by the basic reproduction term $R_{0}$. If $R_{0}<1$, the disease-free (zero) equilibrium will be asymptotically stable. On the other hand, if $R_{0}>1$, there exists a positive equilibrium that is globally/orbitally asymptotically stable under certain conditions within the interior of a predefined region. Finally, numerical simulations are conducted to illustrate and verify the results.
\end{abstract}

\section{INTRODUCTION}

In the field of epidemiology, although our knowledge of viral dynamics and virusspecific immmune responses has not fully developed, numerous mathematical models have been developed an investigated to describe the immunological response to HIV infection (for example, 11, 2, 4, 18, 19, 12, and references therein). The models have been used to explain different phenomena within the host body, and by directly applying the models to real clinical data, they can also predict estimates of many measures, including the death rate of productively infected cells, the rate of viral clearance or the viral production rate.

These simple HIV models have played an essential role in providing a better understanding in the dynamics of this infectious diseases, while providing very important biological meanings for the (combined) drug therapies used against it. For more references and detailed meta mathematical analysis on these models in general, we can refer to survey papers written by Kirschner, 1996 [14] or Perelson and Nelson, 1999 [8]

2020 Mathematics Subject Classification. Primary: 34D23 ; Secondaries: 34D20, 34D05, 34D08.

Key words and phrases. HIV; globally asymptotical stability; periodic solution; delay term; steady state.

(C)2019 Maltepe Journal of Mathematics.

Submitted on April 8th, 2021. Published on April 30th, 2021. Communicated by İbrahim ÇANAK and Sefa Anıl SEZER.. 
The simplest HIV model, only considering the dynamics of the virus concentration, is

$$
\frac{d V}{d t}=P-c V
$$

where

- $P$ is an unknown function representing the rate of production of the virus,

- $V$ is the virus concentration.

The dynamics of the population of target cells $\left(\mathrm{CD} 4^{+}\right.$T-cells for HIV or hepatic cells for HBV and HCV) is still not fully understood. Nevertheless, a reasonable, simple model for this population of cells, which can be extended further in various models, is

$$
\frac{d T}{d t}=s-d T+a T\left(1-\frac{T}{T_{\max }}\right)
$$

with

- $s$ representing the rate at which new T-cells are created from sources within the body, such as the thymus, or from the proliferation of existing T-cells,

- $d$ being the death rate per T-cells,

- $a$ is the maximum proliferation rate of target T-cells, when the proliferation is represented by a logistic function, and

- $T_{\max }$ is the population density of T-cells at which proliferation shuts off.

Human immunodeficiency virus, or HIV, is a virus belonging to the genus Lentivirus, part of the family Retroviridae [27]. It has an outer envelope of lipid and viral proteins, which encloses its core. The virion core contains two positive-sense single-stranded RNA and the enzyme reverse transcriptase, an RNA-dependent DNA polymerase.

HIV, like most viruses, cannot reproduce by itself. Therefore, they require a host cell and its materials to replicate. For HIV, it infects a variety of immune cells, including helper $\mathrm{T}$ cells, lymphocytes, monocytes, and dendritic cells by attaching to a specific receptor called the CD4 receptor contained in the cell membrane. Along with a chemokine coreceptor, the virus is granted entry into the cell. Inside the host cell, the viral RNA is transcribed into DNA by the enzyme reverse transcriptase. However, the enzyme has no proofreading capacity, so errors often occur during this process, giving rise to 1 to 3 mutations per newly synthesized virus particle. The DNA provirus is then transported into the nucleus and inserts itself into the host cell DNA with the aid of viral integrase. Thus, the viral genetic code becomes a stable part of the cell genome, which is then transcribed into a full-length mRNA by the host cell RNA polymerase. The full-length mRNA would be

(1) the genomes of progeny virus, which would be transported to the cytoplasm for assembly,

(2) translated to produce the viral proteins, including reverse transcriptase and integrase, and

(3) spliced, creating new translatable sequences

The nonstructural genes on the virus also encode regulatory proteins that have diverse effects on the host cell, including down-regulating host cell receptors like CD4 and major histocompatibility complex class I molecules, aiding in synthesizing full-length HIV RNAs and enabling transportation of the viral mRNAs out of the 
nucleus without being spliced by the host cell. Altogether, these effects enable viral mRNAs to be correctly translated into polypeptides and packaged into virions. These components are then transported to the plasma membrane and assembled into the mature virion, exiting the cell.

A person can contract the virus through one of four routes: sexual contact, either homo- or heterosexual; transfusions with whole blood, plasma, clotting factors and cellular fractions of blood; contaminated needles; perinatal transmission. The virus causes tissue destruction, immunodeficiency and can progress to acquired immunodeficiency syndrome (AIDS), completely breaking down the human body's defense mechanisms. These patients are now more susceptible to infections that should be harmless to a normal person, such as P.jiroveci pneumonia or tuberculosis, and the conditions are worse as well. So far, treatments for the disease mainly target reverse transcriptase, viral proteases, and viral integration and fusion, dealing with the virus infection before it progresses to AIDS. Currently, one treatment for HIV is highly active antiretroviral therapy (HAART), which includes a combination of drugs including nucleoside/nucleotide analog reverse transcriptase inhibitors, nonnucleoside reverse transcriptase inhibitors, protease inhibitors, fusion inhibitors, integrase inhibitors, and coreceptor blockers. These drugs are administered based on individualized criteria such as tolerability, drug-drug interactions, convenience/adherence, and possible baseline resistance. Although HAART can lower the viral load, the virus reemerges if the treatment is stopped. Therefore, HIV infection is currently both chronic and incurable. 28.

Whenever the population reaches $T_{\max }$, it will decrease, allowing us to impose an upper constrain $d T_{\max }<s$. With this constrain, the equation 1.2 has a unique equilibrium at

$$
\hat{T}=\frac{T_{\max }}{2 a}\left[a-d+\sqrt{(a-d)^{2}+\frac{4 a s}{T_{\max }}}\right]
$$

In 1989, Perelson [5] proposed a general model for the interaction between the human immune system and HIV; in the same paper, he also simplified that general model into a simpler model with four compartments, whose dynamics are described by a system of four ODEs:

- Concentration of cells that are uninfected $(T)$,

- Concentration of cells that are latently infected $\left(T^{*}\right)$,

- Concentration of cells that are actively infected $\left(T^{* *}\right)$, and

- Concentration of free infectious virus particles $(v)$.

Later, he extended his own model in Perelson et al. (1993) [6] by proving various mathematical properties of the model, choosing parameter values from a restricted set that give rise to the long incubation period characteristic of HIV infection, and presenting some numerical solutions. He also observed that his model exhibits many clinical symptoms of AIDS, including:

- Long latency period,

- Low levels of free virus in the environment, and

- Depletion of $\mathrm{CD} 4^{+}$cells.

The paper will be organized as follows: First, we will investigate a simplified ODE model from Perelson et al. (1993) [6] by considering three main components: the uninfected CD4 ${ }^{+}$T-cells $(T)$, the infected CD4 ${ }^{+}$T-cells $(I)$, and the free virus $(V)$ with. This model is also assumed to have a saturation response of the infection 
rate. Next, the existence and stability of the infected steady state are considered through different theorems. Finally, numerical simulations are carried out, using Julia, to confirm the obtained results, before some remarks are included in the conclusion.

\section{The proposal of the ODE Model}

Simplifying the model proposed in Perelson et al. (1993) [6] by reducing the number of dimensions and assuming that all of the infected cells have the ability of producing virus at an equal rate, we propose the following epidemic model of HIV infection of $\mathrm{CD} 4^{+}$T-cells as follows:

$$
\begin{aligned}
\frac{d T}{d t} & =s-d T+a T\left(1-\frac{T}{T_{\max }}\right)-\frac{\beta T V}{1+\alpha V}+\rho I \\
\frac{d I}{d t} & =\frac{\beta T V}{1+\alpha V}-(\delta+\rho) I \\
\frac{d V}{d t} & =q I-c V-k_{1} V T
\end{aligned}
$$

where

- $T(t)$ is the concentration of healthy $\mathrm{CD} 4^{+} \mathrm{T}$-cells at time $t$ (target cells),

- $I(t)$ is the concentration of infected $\mathrm{CD} 4^{+} \mathrm{T}$-cells at time $t$, and

- $V(t)$ is the viral load of the virions (concentration of free HIV at time $t$ ).

In infection modelling, it is very common to augment 2.1] with a "mass-action" term in which the rate of infection is given by $\beta T V$. This type of term is sensible, since the virus must interact with T-cells in order to infect and the probability of virus encountering a T-cell at a low concentration environment (where infected cells and viral load's motions are regarded as independent) can be assumed to be proportional to the product of the density, which is called linear infection rate. As a result, it follows that the classical models can assume that T-cells are infected at rate $-\beta T V$ and are generated at rate $\beta T V$.

With that simple mass-action infection term, the rates of change of uninfected cells, $T$, productively infected cells $I$, and free virus $V$, would be

$$
\begin{aligned}
\frac{d T}{d t} & =s-d T+a T\left(1-\frac{T}{T_{\max }}\right)-\beta T V \\
\frac{d I}{d t} & =\beta T V-\delta I \\
\frac{d V}{d t} & =q I-c V
\end{aligned}
$$

Moreover, although the rate of infection in most HIV models is bilinear for the virus $V$ and the uninfected target cells $T$, the actual incidence rates are probably not strictly linear for each variable in over the whole valid range. For example, a non-linear or less-than-linear response in $V$ could occur due to the saturation at a high enough viral concentration, where the infectious fraction is significant for exposure to happen very likely. Thus, is it reasonable to assume that the infection rate of HIV modelling in saturated mass action is

$$
\frac{\beta T V^{x}}{1+\alpha V^{y}}, \quad x, y, \alpha>0
$$


In this paper, we will investigate the viral model with saturation response of the infection rate where $x=y=1$, for the sake of simplicity. With that being said, we will proceed to explain the parameters within the model, with

- $s$ is the rate at which new T-cells are created from source from precursors,

- $d$ is the natural death rate of the $\mathrm{CD} 4^{+} \mathrm{T}$-cells,

- $a$ is the maximum proliferation rate (growth rate) of T-cells (this means that $a>d$, in general),

- $T_{\max }$ is the T-cells population density at which proliferation shuts off (their carrying capacity),

- $\beta$ is the rate constant of infection of T-cells with free virus,

- $\rho$ is the "cure" rate, or the non-cytolytic loss of infected cells,

- $\delta$ is the death rate of the infected cells,

- $q$ is the reproduction rate of the infected cells, and

- $c$ is the clearance rate constant (loss rate) of the virions.

From the explanations above, we can say that

- $\delta+\rho$ is the total rate of disappearance of infected cells from the environment,

- $1 / \delta$ is the average lifespan of a productively infected cell

- $q / \delta$ is the total number of virions produced by an actively infected cell during its lifespan, and

- $q$ is the average rate of virus released by each cell.

Under the absence of virus (i.e, $I(t)=V(t)=0 \quad \forall t>0$ ), the T-cell population has a steady state value of

$$
T_{0}=\frac{T_{\max }}{2 a}\left[(a-d)+\sqrt{(a-d)^{2}+\frac{4 a}{T_{\max }}}\right]
$$

The system 2.1 needs to be initialized with the following initial conditions

$$
T(0)>0, \quad I(0)>0, \quad V(0)>0,
$$

which lead us to denote that

$$
R_{+}^{3}=\left\{(T, I, V) \in \mathbb{R}^{3} \| T \geq 0, I \geq 0, V \geq 0\right\} .
$$

\section{Equilibrium And Stability of the PROposed MOdel}

3.1. Equilibria and local stability. The system 2.1 has two steady states: the uninfected steady state $E_{0}=\left(T_{0}, 0,0\right)$ and the (positive) infected steady state $\bar{E}=(\bar{T}, \bar{I}, \bar{V})$, where:

$$
\begin{aligned}
& \bar{T}=\frac{T_{\max }}{2 a}\left[a-d-\delta \frac{q \beta-(\delta+\rho)}{q \alpha(\delta+\rho)}+\sqrt{\left(a-d-\delta \frac{q \beta-(\delta+\rho)}{q \alpha(\delta+\rho)}\right)^{2}-\frac{4 a}{T_{\max }}\left(\frac{\delta c}{q \alpha}-s\right)}\right. \\
& \bar{I}=\frac{\left[q \beta-(\delta+\rho) k_{1}\right] \bar{T}-(\delta+\rho) c}{q \alpha(\delta+\rho)} \\
& \bar{V}=\frac{1}{\alpha}\left[\frac{q \beta \bar{T}}{\alpha(\delta+\rho)\left(c_{1}+k_{1} T\right.}-1\right] .
\end{aligned}
$$

Now, we will proceed to analyse the stability of the equilibria of system 2.1 . 
Since $T_{0}$ and $\bar{T}$ satisfy

$$
\begin{aligned}
s-d T_{0}+a T_{0}\left(1-\frac{T_{0}}{T_{\max }}\right) & =0 \\
s-d \bar{T}+a \bar{T}\left(1-\frac{\bar{T}}{T_{\max }}\right) & =\delta \bar{I}=\frac{\delta}{q \alpha(\delta+\rho)}[(q \beta-(\delta+\rho)) T-(\delta+\rho) c]
\end{aligned}
$$

we get that

$\bar{T}>\frac{c(\delta+\rho)}{q \beta-(\delta+\rho) k_{1}} \Rightarrow s-d \bar{T}+a \bar{T}\left(1-\frac{\bar{T}}{T_{\max }}\right)>0 \quad \Rightarrow \quad T_{0}>\bar{T}$,

and

$\bar{T}<\frac{c(\delta+\rho)}{q \beta-(\delta+\rho) k_{1}} \quad \Rightarrow \quad s-d \bar{T}+a \bar{T}\left(1-\frac{\bar{T}}{T_{\max }}\right)<0 \quad \Rightarrow \quad T_{0}<\bar{T}$.

Hence,

- If $\bar{T}>\frac{c(\delta+\rho)}{q \beta-(\delta+\rho) k_{1}}$, then $T_{0}>\bar{T}>\frac{c(\delta+\rho)}{q \beta-(\delta+\rho) k_{1}}$, which means that $E_{0}\left(T_{0}, 0,0\right)$ is unstable, while the positive equilibrium $\bar{E}(\bar{T}, \bar{I}, \bar{V})$ exists.

- If $\bar{T}<\frac{c(\delta+\rho)}{q \beta-(\delta+\rho) k_{1}}$, then $T_{0}<\bar{T}<\frac{c(\delta+\rho)}{q \beta-(\delta+\rho) k_{1}}$, which means that $E_{0}\left(T_{0}, 0,0\right)$ is locally asymptotically stable, while the positive equilibrium $\bar{E}(\bar{T}, \bar{I}, \bar{V})$ is not feasible, as $\bar{I}<0, \bar{V}<0$.

Let

$$
R_{0}=\left(\frac{q \beta-(\delta+\rho) k_{1}}{c(\delta+\rho)}\right) \bar{T} .
$$

We can see that $R_{0}$ is the bifurcation parameter. When $R_{0}<1$, the uninfected steady state $E_{0}$ is stable and the infected steady state $\bar{E}$ does not exist (unphysical). When $R_{0}>1, E_{0}$ becomes unstable and $\bar{E}$ exists.

For system 2.2 , it is known that the basic reproductive ratio is given by:

$$
R_{01}=\left(\frac{q \beta-(\delta+\rho) k_{1}}{c(\delta+\rho)}\right) T_{0}
$$

Once again, we emphasize the large difference of the basic reproduction ratio between the linear infection rate and the saturation infection rate.

- If $\alpha \rightarrow 0$, then $\bar{T} \rightarrow \frac{c(\delta+\rho)}{q \beta-(\delta+\rho)}, \quad R_{0} \rightarrow 1$;

- If $\alpha \rightarrow+\infty$, then $\bar{T} \rightarrow T_{0}, R_{0} \rightarrow R_{01}$.

The Jacobian matrix of system (2.1) is:

$$
\left(\begin{array}{ccc}
(a-d)-\frac{2 a T}{T_{\max }}-\frac{\beta V}{1+\alpha V} & \rho & -\frac{\beta T}{(1+\alpha V)^{2}} \\
\frac{\beta V}{1+\alpha V} & -(\delta+\rho) & \frac{\beta T}{(1+\alpha V)^{2}} \\
-k_{1} V & q & -c-k_{1} T
\end{array}\right) .
$$

Let $E^{*}\left(T^{*}, I^{*}, V^{*}\right)$ be any arbitrary equilibrium. Then, the characteristic equation about $E^{*}$ is: 


$$
\left|\begin{array}{ccc}
\lambda+\left((d-a)+\frac{2 a T^{*}}{T_{\max }}+\frac{\beta V^{*}}{1+\alpha V^{*}}\right) & -\rho & \frac{\beta T^{*}}{\left(1+\alpha V^{*}\right)^{2}} \\
-\frac{\beta V^{*}}{1+\alpha V^{*}} & \lambda+(\delta+\rho) & -\frac{\beta T^{*}}{\left(1+\alpha V^{*}\right)^{2}} \\
k_{1} V^{*} & -q & \lambda+\left(c+k_{1} T^{*}\right)
\end{array}\right|=0 .
$$

For equilibrium $E_{0}=\left(T_{0}, 0,0\right)$, 3.8 reduces to

$$
\left(\lambda-a+d+\frac{2 a T_{0}}{T_{\max }}\right)\left[\lambda^{2}+(c+\delta+\rho) \lambda+c(\delta+\rho)-q \beta T_{0}\right]=0
$$

Hence, we can see that $E_{0}=\left(T_{0}, 0,0\right)$ is locally asymptotically stable if $R_{0}<1$, and it is a saddle point if $\operatorname{dim} W^{s}\left(E_{0}\right)=2$, or if $\operatorname{dim} W^{s}\left(E_{0}\right)=1$ while $R_{0}>1$. As a result, we have the following theorems.

Theorem 3.1. If $R_{0}<1, E_{0}=\left(T_{0}, 0,0\right)$ is locally asymptotically stable; else, if $R_{0}>1, E_{0}=E_{0}=\left(T_{0}, 0,0\right)$ is unstable.

Theorem 3.2. There exists $M>0, M \in \mathbb{R}$ such that for any positive solution $(T(t), I(t), V(t))$ of system 2.1),

$$
T(t) \leq M, I(t) \leq M, V(t) \leq M
$$

for all large enough $t$.

Proof. Let $L(t)=T(t)+I(t)$ and assume that $L(0)=T(0)+I(0)=$ const $=c$. Calculating the derivative of $L(t)$ using the equations in system (2.1), we have:

$$
\begin{aligned}
\frac{d L(t)}{d t} & =\frac{d T(t)}{d t}+\frac{d I(t)}{d t} \\
& =s-d T+a T\left(1-\frac{T}{T_{\max }}\right)-\delta I \\
& =-d t-\delta I-\frac{a}{T_{\max }}\left(T-\frac{T_{\max }}{2 a}\right)^{2}+\frac{4 s+a T_{\max }}{4} \\
& \leq-(T+I) \min (d, \delta)-\frac{a}{T_{\max }}\left(T-\frac{T_{\max }}{2 a}\right)^{2}+\frac{4 s+a T_{\max }}{4} \\
& =-h L(t)-M_{0}\left(h=\min (d, \delta), M_{0}=\frac{4 s+a T_{\max }}{4}\right)
\end{aligned}
$$

Let $U(t)=L(t)-\frac{M_{0}}{h}$. This means that

$$
\begin{aligned}
U(0) & =L(0)-\frac{M_{0}}{h}=c-\frac{M_{0}}{h} \\
\frac{d U(t)}{d t} & =\frac{d L(t)}{d t}
\end{aligned}
$$

The inequality (3.11) can be rewritten as

$$
\frac{d U(t)}{d t} \leq(-h) U(t)
$$

which yields, according to Gronwall's inequality, 


$$
\begin{aligned}
U(t) & \leq U(0) \exp \left(\int_{0}^{t}(-h) d s\right) \\
& =\left(c-\frac{M_{0}}{h}\right) \exp \left([-h s]_{0}^{t}\right) \\
& =\left(c-\frac{M_{0}}{h}\right) \exp (-h t) \\
& \leq c-\frac{M_{0}}{h}
\end{aligned}
$$

or

$$
T(t)+I(t)=L(t)=U(t)+\frac{M_{0}}{h}=c-\frac{M_{0}}{h}+\frac{M_{0}}{h}=c .
$$

As $T(t)>0, I(t)>0 \forall i \in \mathbb{Z}^{+}$, we can say that

$$
V(t) \leq c, \quad I(t) \leq c .
$$

Moreover, we also know that

$$
\frac{d V}{d t}=q I-c V-k_{1} V T \leq q I-c V \leq q c-c V=-c(V-q) .
$$

Setting $V(0)=$ const $=c_{V}$, using the exact same procedure with Gronwall's inequality, we obtain

$$
V(t) \leq c_{V} \quad \forall t \in \mathbb{Z}^{+}
$$

With $M=\max \left(c, c_{V}\right)$, we would then conclude that

$$
T(t) \leq M, \quad I(t) \leq M, \quad V(t) \leq M \forall t \in \mathbb{Z}^{+} .
$$

We can easily see that this set is convex. As a consequence, the system (2.1) is dissipative.

The proof is complete.

From this theorem, we define

$$
D=\left\{(T, I, V) \in \mathbb{R}^{3}, 0 \leq T, I, V \leq M\right\}
$$

Denote

$$
M=d-a+\frac{2 a \bar{T}}{T_{\max }}, \quad N=\frac{\beta \bar{V}}{1+\alpha \bar{V}}, \quad P=\frac{\beta \bar{T}}{(1+\alpha \bar{V})^{2}} .
$$

Then, the characteristic equation of the system around the equilibrium $\bar{E}(\bar{T}, \bar{I}, \bar{V})$ reduces to:

$$
\lambda^{3}+a_{1} \lambda^{2}+\left(a_{2}+a_{4}\right) \lambda+\left(a_{3}+a_{5}\right)=0
$$

where 


$$
\begin{aligned}
& a_{1}=M+\left(\delta+\rho+c_{1}+k_{1} \bar{T}\right) \\
& a_{2}=(\delta+\rho)\left(c_{1}+k_{1} T\right)+M\left(\delta+\rho+c_{1}+k_{1} \bar{T}\right)+\left(-k_{1} \bar{V} P\right) \\
& a_{3}=\rho\left[-N\left(c_{1}+k_{1} \bar{T}\right)+P k_{1} \bar{V}\right]+P N q \\
& a_{4}=-N P \\
& a_{5}=M(\delta+\rho)\left(c_{1}+k_{1} \bar{T}\right)-P(\delta+\rho) k_{1} \bar{V} .
\end{aligned}
$$

By the Routh-Hurwitz criterion [15, it follows that all eigenvalues of equation (3.22) have negative real parts if and only if

$$
a_{1}>0, \quad a_{3}+a_{5}>0, \quad a_{1}\left(a_{2}+a_{4}\right)-\left(a_{3}+a_{5}\right)>0 .
$$

This leads us to the following theorems.

Theorem 3.3. Suppose that

(1) $R_{0}>1$

(2) $a_{1}>0, \quad a_{3}+a_{5}>0, \quad a_{1}\left(a_{2}+a_{4}\right)-\left(a_{3}+a_{5}\right)>0$.

Then, the positive equilibrium $\bar{E}(\bar{T}, \bar{I}, \bar{V})$ is asymptotically stable.

Theorem 3.4. If $R_{0}<1$, then $E_{0}\left(T_{0}, 0,0\right)$ is globally asymptotically stable.

Proof. First of all, as $R_{0}<1$, we would have

$$
T_{0}<\bar{T}<\frac{c(\delta+\rho)}{q \beta-(\delta+\rho)}
$$

which means that

$$
p<\frac{\left(c+k_{1} T\right)(\delta+\rho)}{\beta T} .
$$

From the system (2.1), we would have

$$
\begin{aligned}
\frac{d I}{d t} & \leq \beta T V-(\delta+\rho) I \\
\frac{d V}{d t} & =q I-c V-k_{1} V T .
\end{aligned}
$$

Now, we would consider the following comparative system

$$
\begin{aligned}
& \frac{d z_{1}}{d t}=\beta T z_{2}-(\delta+\rho) z_{1} \\
& \frac{d z_{2}}{d t}=p z_{1}-c z_{2}-k_{1} z_{2} T .
\end{aligned}
$$

We will consider the following form of Lyapunov function:

$$
L(\mathbf{X})=V\left(z_{1}, z_{2}\right)=\frac{\delta+\rho}{(\beta T)^{2}} z_{1}^{2}+\frac{1}{c+k_{1} T} z_{2}^{2} .
$$

The derivative of the function can be calculated as follows 


$$
\begin{aligned}
\frac{d L}{d t} & =\frac{\partial L}{\partial z_{1}} \frac{d z_{1}}{d t}+\frac{\partial L}{\partial z_{2}} \frac{d z_{2}}{d t} \\
& =2 \frac{\delta+\rho}{(\beta T)^{2}} z_{1}\left(\beta T z_{2}-(\delta+\rho) z_{1}\right)+2 \frac{1}{c+k_{1} T} z_{2}\left(q z_{1}-c z_{2}-k_{1} T z_{2}\right) \\
& =-2\left[\left(\frac{\delta+\rho}{\beta T} z_{1}\right)^{2}+z_{2}^{2}-\left(\frac{\delta+\rho}{\beta T} z_{1} z_{2}+\frac{q}{c+k_{1} T}\right) z_{1} z_{2}\right] \\
& \leq-2\left[\left(\frac{\delta+\rho}{\beta T} z_{1}\right)^{2}+z_{2}^{2}-\left(\frac{\delta+\rho}{\beta T}+\frac{\beta+\rho}{\beta T}\right) z_{1} z_{2}\right] \\
& =-2\left[\frac{\delta+\rho}{\beta T} z_{1}-z_{2}\right]^{2} \leq 0 \quad \forall z_{1}, z_{2} .
\end{aligned}
$$

We can see that the derivative is negative definite everywhere except at $(0,0)$. This means that $\left(z_{1}, z_{2}\right)=(0,0)$ is globally asymptotically stable.

As we can also see that

$$
0 \leq I(0) \leq z_{1}(0), \quad 0 \leq V(0) \leq z_{2}(0)
$$

which means that, if the system 3.28 admits the initial values $\left(z_{1}(0), z_{2}(0)\right)$, we have that

$$
I(t) \leq z_{1}(t), \quad V(t) \leq z_{2}(t) \quad \forall t>t_{1}
$$

or, in other words,

$$
\lim _{t \rightarrow+\infty} I(t)=\lim _{t \rightarrow+\infty} V(t)=0 .
$$

From this, using the first equation of the system $(2.1)$, for an $\epsilon$ in $(0,1)$ infinitesimal,

$$
s+(a-d-\delta \epsilon) T-\frac{a T^{2}}{T_{\max }} \leq \frac{d T(t)}{d t} \leq s+(a-d) T-\frac{a T^{2}}{T_{\max }} \quad \forall t>t_{2}
$$

which shows that

$$
\lim _{t \rightarrow+\infty} T(t)=T_{0}
$$

From (3.33) and (3.35), we conclude that the system is globally asymptotically stable. The proof is complete.

Theorem 3.5. If $R_{0}>1$, then the system 2.1] is permanent.

Proof. If $R_{0}>1$, we would have

$$
\left(q \beta-(\delta+\rho) k_{1}\right) T_{0}>\left(q \beta-(\delta+\rho) k_{1}\right) \bar{T}>c(\delta+\rho)
$$

We will proceed to prove the weak permanence of this system using contradiction.

Assume that the system is not weakly permanent, from Theorem 3.4 there exists a positive orbit $(T(t), I(t), V(t))$ such that

$$
\lim _{t \rightarrow+\infty} T(t)=T_{0}, \quad \lim _{t \rightarrow+\infty} I(t)=\lim _{t \rightarrow+\infty} V(t)=0 .
$$


Since $T_{0}>\frac{c(\delta+\rho)}{q \beta-(\delta+\rho)}$, combining with 3.37 , we choose an arbitrary infinitesimal $\epsilon>0$ such that there exists a $t_{0}>0$, for all $t>t_{0}$,

$$
\begin{aligned}
\frac{T_{0}-\epsilon}{1+\alpha \epsilon} & >\frac{c(\delta+\rho)}{q \beta-(\delta+\rho)} \\
T(t) & >T_{0}-\epsilon, \\
V(t) & <\epsilon .
\end{aligned}
$$

Under these conditions, the system (2.1) becomes

$$
\begin{aligned}
\frac{d I}{d t} & =\frac{\beta T V}{1+\alpha V}-(\delta+\rho) I \geq \frac{\beta\left(T_{0}-\epsilon\right)}{1+\alpha \epsilon} V-(\delta+\rho) I(t) \\
\frac{d V}{d t} & =q I-\left(c_{1}+k_{1} T\right) \approx q I-c V-k_{1} T_{0}
\end{aligned}
$$

Consider the following Jacobian matrix

$$
J_{\epsilon}=\left(\begin{array}{cc}
-(\delta+\rho) & \frac{\beta\left(T_{0}-\epsilon\right)}{1+\alpha \epsilon} \\
q & -\left(c+k_{1} T_{0}\right)
\end{array}\right)
$$

Since $J_{\epsilon}$ has positive off-diagonal element, according to the Perron - Frobenius theorem, for the maximum positive eigenvalue $j_{1}$ of $J_{\epsilon}$, there is an associated positive eigenvector $v=\left(\begin{array}{l}v_{1} \\ v_{2}\end{array}\right)$.

Next, we consider a system associated with the Jacobian matrix $J_{\epsilon}$

$$
\begin{aligned}
\frac{d z_{1}}{d t} & =\frac{\beta\left(T_{0}-\epsilon\right)}{1+\alpha \epsilon} z_{2}-(\delta+\rho) z_{1} \\
\frac{d z_{2}}{d t} & =q z_{1}-\left(c+k_{1} T_{0}\right) z_{2} .
\end{aligned}
$$

Let $z(t)=\left(z_{1}(t), z_{2}(t)\right)$ be a solution of 3.41 through $\left(l v_{1}, l v_{2}\right)$ at $t=t_{0}$, where $l>0$ satisfies that

$$
l v_{1}<I\left(t_{0}\right), \quad l v_{2}<V\left(t_{0}\right) .
$$

As we know that the semi-flow of 3.41 is monotone and $J_{\epsilon} v=v>0, z_{i}(t)(t=$ $1,2)$ is strictly increasing, meaning $\lim _{t \rightarrow+\infty} z_{i}(t)=+\infty$. This contradicts the Theorem 3.2 saying that the positive solution of 2.1 is bounded from above. This contradiction says that there exists no positive orbit of 2.1 tends to $\left(T_{0}, 0,0\right)$ and $t \rightarrow+\infty$. Combining this and a result provided in [23], we conclude that the system 2.1 is permanent.

The proof is complete.

Theorem 3.6. Assume that $D$ is convex and bounded. Suppose that the system

$$
\frac{d X}{d t}=F(X), \quad X \in D
$$

is competitive, permanent and has the property of stability of periodic orbits. If $\bar{X}_{0}$ is the only equilibrium point in intD and if it is locally asymptotically stable, then it is globally asymptotically stable in intD. 
Proof. This matrix can easily be proven by considering the Jacobian matrix and choose the matrix $H$ as

$$
H=\left(\begin{array}{ccc}
1 & 0 & 0 \\
0 & -1 & 0 \\
0 & 0 & 1
\end{array}\right)
$$

By simple calculation, we obtain that

$$
H \frac{\partial f}{\partial x} H=\left(\begin{array}{ccc}
(a-d)-\frac{2 a T}{T_{\max }}-\frac{\beta V}{1+\alpha V} & -\rho & -\frac{\beta T}{(1+\alpha V)^{2}} \\
-\frac{\beta V}{1+\alpha V} & -(\delta+\rho) & -\frac{\beta T}{(1+\alpha V)^{2}} \\
-k_{1} V & -q & -c-k_{1} T
\end{array}\right) .
$$

This means that the system 2.1 is competitive in $D$, with respect to the partial order defined by the orthant

$$
K=\left\{(T, I, V) \in \mathbb{R}^{3} \| T \leq 0, I \geq 0, V \geq 0\right\} .
$$

Remark. As D is convex and the system (2.1) is competitive in D, we can say that the system (2.1) satisfies the Poincare - Bendixson property. This has been proven by Hirsch (1990) 22], Zhu and Smith (1994) 21] and Smith and Thieme (1991) 24 that any three-dimensional competitive system that lie in convex sets would have the Poincaré - Bendixson property; in other words, any non-empty compact omega limit set that contains no equilibria must be a closed orbit.

Theorem 3.7. Let $c=I(0)+T(0)$ and suppose that

(1) $R_{0}>1$

(2) $a_{1}>0, a_{3}+a_{5}>0, a_{1}\left(a_{2}+a_{4}\right)-\left(a_{3}+a_{5}\right)>0$.

Then, the positive equilibrium $\bar{E}(\bar{T}, \bar{I}, \bar{V})$ of system (2.1) is globally asymptotically stable provided that one of the following two assumptions hold

(3) $T_{\max } \frac{a-d+k_{1} c}{2 a}<m<T_{0}<T_{\max } \frac{a-d+\delta+k_{1} c}{2 a}$,

(4) $m>T_{\max } \frac{a-d+\delta+k_{1} c}{2 a}$.

As we have already known that the system $(2.1)$ is competitive and permanent (from Theorem 3.5 and Theorem 3.6 , while $E(T, \bar{I}, \bar{V})$ is locally asymptotically stable if the two properties (i) and (ii) of Theorem 3.7 holds. As a result, in accordance with Theorem 3.6 (choosing $D=\Omega$ ), Theorem 3.7 if we can prove that the system 2.1 has the stability of periodic orbits. We will proceed to prove this under the following proposition.

Proposition 3.8. Assume condition 3. or 4. of Theorem 3.7 hold true. Then, system (2.1) has the property of stability of periodic orbits.

Proof. Let $P(t)=((T(t), I(t), V(t))$ be a periodic solution whose orbit $\Gamma$ is contained in int $\Omega$. In accordance with the criterion given by Muldowney in [25, for the asymptotic orbital stability of a periodic orbit of a general autonomous system, it is sufficient to prove that the linear non-autonomous system

$$
\frac{d W(t)}{d t}=\left(D F^{[2]}(P(t))\right) W(t)
$$

is asymptotically stable, where $D F^{[2]}$ is the second additive compound matrix of the Jacobian $D F[1]$. 
The Jacobian matrix of the system 2.1) is given by

$$
J=\left(\begin{array}{ccc}
(a-d)-\frac{2 a T}{T_{\max }}-\frac{\beta V}{1+\alpha V} & \rho & -\frac{\beta T}{(1+\alpha V)^{2}} \\
\frac{\beta V}{1+\alpha V} & -(\delta+\rho) & \frac{\beta T}{(1+\alpha V)^{2}} \\
-k_{1} V & q & -\left(c+k_{1} T\right)
\end{array}\right) .
$$

For the solution $P(t)$, the equation (3.47) becomes

$$
\begin{aligned}
\frac{d W_{1}}{d t} & =-\left(\delta+\rho-(a-d)+\frac{2 a T}{T_{\max }}+\frac{\beta V}{1+\alpha V}\right) W_{1}+\frac{\beta T}{(1+\alpha V)^{2}}\left(W_{2}+W_{3}\right), \\
\frac{d W_{2}}{d t} & =q W_{1}+\left(a-d-\frac{2 a T}{T_{\max }}-\frac{\beta V}{1+\alpha V}-\left(c+k_{1} T\right)\right) W_{2}+\rho W_{3} \\
\frac{d W_{3}}{d t} & =k_{1} V W_{1}+\frac{\beta V}{1+\alpha V} W_{2}-\left(\delta+\rho+c+k_{1} T\right) W_{3} .
\end{aligned}
$$

To prove that the system 3.49 is asymptotically stable, we shall use the following Lyapunov function, which is similar to the one found in [26] for the SEIR model:

$$
L\left(W_{1}(t), W_{2}(t), W_{3}(t), T(t), I(t), V(t)\right)=\left\|\left(W_{1}(t), \frac{I(t)}{V(t)} W_{2}(t), \frac{I(t)}{V(t)} W_{3}(t)\right)\right\|,
$$

where $\|\cdot\|$ is the norm in $\mathbb{R}^{3}$ defined by

$$
\left\|\left(W_{1}, W_{2}, W_{3}\right)\right\|=\sup \left\{\left|W_{1}\right|,\left|W_{2}+W_{3}\right|\right\} .
$$

From Theorem 3.5. we obtain that the orbit of $P(t)$ remains at a positive distance from the boundary of $\Omega$. Therefore,

$$
I(t) \geq \eta, \quad V(t) \geq \eta, \quad \eta=\min \{\underline{\mathrm{I}}, \underline{\mathrm{V}}\} \quad \forall t \rightarrow+\infty .
$$

Hence, the function $L(t)$ is well defined along $P(t)$ and

$$
L\left(W_{1}, W_{2}, W_{3} ; T, I, V\right) \geq \frac{\eta}{M}\left\|\left(W_{1}, W_{2}, W_{3}\right)\right\| .
$$

Along a solution $\left(W_{1}, W_{2}, W_{3}\right)$ of the system $(3.49), L(t)$ becomes

$$
L(t)=\sup \left\{\left|W_{1}(t)\right|, \frac{I(t)}{V(t)}\left(\left|W_{2}(t)\right|+\left|W_{3}(t)\right|\right)\right\} .
$$

Then, we would have the following inequalities

$$
\begin{aligned}
& D_{+}\left|W_{1}(t)\right| \leq-\left(\delta+\rho-(a-d)+\frac{2 a T}{T_{\max }}+\frac{\beta V}{1+\alpha V}\right)\left|W_{1}\right|+\frac{\beta T}{(1+\alpha V)^{2}}\left(\left|W_{2}(t)\right|+\left|W_{3}(t)\right|\right) \\
& D_{+}\left|W_{2}(t)\right| \leq q\left|W_{1}(t)\right|+\left(a-d-\frac{2 a T}{T_{\max }}-\frac{\beta V}{1+\alpha V}-\left(c+k_{1} T\right)\right)\left|W_{2}(t)\right|+\rho\left|W_{3}(t)\right| \\
& D_{+}\left|W_{3}(t)\right| \leq k_{1} V\left|W_{1}(t)\right|+\frac{\beta V}{1+\alpha V}\left|W_{2}(t)\right|-\left(\delta+\rho+c+k_{1} T\right)\left|W_{3}(t)\right| .
\end{aligned}
$$

From this, we get 


$$
\begin{aligned}
D_{+} \frac{I}{V}\left(\left|W_{2}\right|+\left|W_{3}\right|\right) & =\left(\frac{d I / d t}{V}-\frac{I d V / d t}{V^{2}}\right)\left(\left|W_{2}\right|+\left|W_{3}\right|\right)+\frac{I}{V} D_{+}\left(\left|W_{2}\right|+\left|W_{3}\right|\right) \\
& \leq\left(\frac{d I / d t}{I}-\frac{d V / d t}{V}\right) \frac{I}{V}\left(\left|W_{2}\right|+\left|W_{3}\right|\right)+\left(\frac{q I}{V}+k_{1} I\right)\left|W_{1}\right| \\
& -\left(-a+d+\frac{2 a T}{T_{\max }}+\left(c+k_{1} T\right)\right) \frac{I}{V}\left|W_{2}(t)\right|-\left(\delta+c+k_{1} T\right) \frac{I}{V}\left|W_{3}(t)\right| .
\end{aligned}
$$

Thus, we can obtain

$$
D_{+} L(t) \leq \sup \left\{g_{1}(t), g_{2}(t)\right\} L(t),
$$

where

$$
\begin{aligned}
g_{1}(t) & =-\delta-\rho+a-d-\frac{2 a T}{T_{\max }}-\frac{\beta V}{1+\alpha V}+\frac{\beta T V}{I(1+\alpha V)^{2}} \\
g_{2}(t) & =\frac{q I}{V}+k_{1} I+\frac{d I / d t}{I}-\frac{d V / d t}{V}-G_{1} \\
G_{1} & =\min \left\{-a+d+\frac{2 a T}{T_{\max }}+\left(c+k_{1} T\right), \delta+c+k_{1} T\right\} .
\end{aligned}
$$

From the second equation of the system (2.1), we obtain

$$
\begin{aligned}
g_{1}(t) & =-\delta-\rho+a-d-\frac{2 a T}{T_{\max }}-\frac{\beta V}{1+\alpha V}+\frac{\beta T V}{I(1+\alpha V)^{2}} \\
& \leq-\delta-\rho+a-d-\frac{2 a T}{T_{\max }}-\frac{\beta V}{1+\alpha V}+\frac{\beta t V}{I(1+\alpha V)} \\
& =a-d-\frac{2 a T}{T_{\max }}-\frac{\beta T}{1+\alpha V}+\frac{d I / d t}{I} .
\end{aligned}
$$

Here, we consider two different cases.

- Case 1: If Point 3. of Theorem 3.7 holds, then

$$
-\delta<a-d-\frac{2 a T}{T_{\max }}<0
$$

that is

$$
G_{1}=-a+d+\frac{2 a T}{T_{m} a x}+\left(c+k_{1} T\right) .
$$

Then, we would obtain

$$
g_{2}(t)=a-d-\frac{2 a T}{T_{\max }}+k_{1} I+\frac{d I / d t}{I}=g_{1}(t)+k_{1} I+\frac{\beta V}{1+\alpha V}>g_{1}(t) .
$$

Hence,

$$
\sup \left\{g_{1}(t), g_{2}(t)\right\} \leq a-d-\frac{2 a T}{T_{\max }}+k_{1} I+\frac{d I / d t}{I} \leq-\mu_{1}+\frac{d I / d t}{I},
$$

where

$$
\mu_{1}>0, \quad a-d-\frac{2 a T}{T_{\max }}+k_{1} I \leq-\mu_{1}<0
$$


with the assumption that $k_{1} I$ is negligible compare to the term $a-\frac{2 a T}{T_{\max }}$. This assumption would be verified in the examples of the simulation part below.

- Case 2: If Point 4. of Theorem 3.7 holds, then

$$
-a+d+\frac{2 a T}{T_{\max }} \leq \delta,
$$

which means that $G_{1}=\delta+c+k_{1} T$. Then, we obtain that

$$
\mu_{2}<0, \quad g_{1}(t)<g_{2}(t)=k_{1} T-\delta+\frac{d I / d t}{I} \leq-\mu_{2}+\frac{d I / d t}{I}
$$

with the same assumption that $k_{1} T<\sigma$ in reasonably practical scenarios. Hence,

$$
\sup \left\{g_{1}(t), g_{2}(t)\right\} \leq-\mu+\frac{d I / d t}{I} .
$$

Let $\mu=\min \left\{\mu_{1}, \mu_{2}\right\}$. Then, form 3.63 and 3.66 , we have

or

$$
\sup \left\{g_{1}(t), g_{2}(t)\right\} \leq-\mu+\frac{d I / d t}{I},
$$

$$
D_{+} L(t) \leq\left(-\mu+\frac{d I / d t}{I}\right) L(t) .
$$

According to Gronwall's inequality, we would have

$$
\begin{aligned}
L(t) & \leq L(0) \exp \left(\int_{0}^{t}\left[-\mu+\frac{d I / d t}{I}\right] d s\right) \\
& =L(0) \exp \left([-\mu s+\ln (I(s))]_{0}^{t}\right) \\
& =L(0) \exp (-\mu t) \exp (\ln (I(t))-\ln (I(0))) \\
& =L(0) \exp (-\mu t) \frac{I(t)}{I(0)} \\
& \leq \frac{M L(0)}{I(0)} \exp (-\mu t) \rightarrow 0 \text { as } t \rightarrow+\infty .
\end{aligned}
$$

From 3.53, we conclude that

$$
\left(W_{1}(t), W_{2}(t), W_{3}(t)\right) \rightarrow 0 \quad \text { as } \quad t \rightarrow+\infty .
$$

This implies that the linear system equation $\sqrt{3.49}$ is asymptotically stable, and, therefore, the periodic solution is asymptotically orbitally stable. This proves proposition 3.8 .

Theorem 3.9. Suppose that

(1) $R_{0}>1$

(2) $a_{1}>0, a_{3}+a_{5}>0, a_{1}\left(a_{2}+a_{4}\right)-\left(a_{3}+a_{5}\right)>0$.

Then, system 2.1 has an orbitally asymptotically stable periodic solution. 
Proof. First, we perform a change of variables as follows:

$$
z_{1}(t)=-T(t), \quad z_{2}(t)=I(t), \quad z_{3}(t)=-V(t)
$$

Applying this transformation to the system 2.1), we obtain

$$
\begin{aligned}
\frac{d z_{1}(t)}{d t} & =-s-d z_{1}+a z_{1}\left(1+\frac{z_{1}}{T_{\max }}\right)+\frac{\beta z_{1} z_{3}}{1-\alpha z_{3}}+\rho z_{2} \\
\frac{d z_{2}(t)}{d t} & =\frac{\beta z_{1} z_{3}}{1-\alpha z_{3}}-(\delta+\rho) z_{2} \\
\frac{d z_{3}(t)}{d t} & =-q z_{2}-c z_{3}+k_{1} z_{1} z_{3} .
\end{aligned}
$$

The Jacobian matrix of the system (3.73) is then given by

$$
J(z)=\left(\begin{array}{ccc}
a-d+\frac{2 a z_{1}}{T_{\max }}+\frac{\beta z_{3}}{1-\alpha z_{3}} & \rho & \frac{\beta z_{1}}{\left(1+\alpha z_{3}\right)^{2}} \\
\frac{\beta z_{3}}{1-\alpha z_{3}} & -(\delta+\rho) & \frac{\beta z_{1}}{\left(1+\alpha z_{3}\right)^{2}} \\
k_{1} z_{3} & -q & -c+k_{1} z_{1} .
\end{array}\right)
$$

Similar to the definition of the set $D$ at 3.20 , we define set $E$ as:

$$
E=\left\{\left(z_{1}, z_{2}, z_{3}\right): z_{1} \leq 0, z_{2} \geq 0, z_{3} \leq 0\right\} .
$$

Since $J(z)$ has non-positive off diagonal elements at each point of $E,(3.73)$ is competitive at $E$. Set $z^{*}=\left(-T^{*}, I^{*}, V^{*}\right)$. It is easy to see that $z^{*}$ is unstable and $\operatorname{det} J\left(z^{*}\right)<0$. Furthermore, it follows from Theorem 3.5 that there exists a compact set $B$ in the interior of $E$ such that for any $z_{0} \in \operatorname{int} E$, there exists $T\left(z_{0}\right)>0$ such that $z\left(t, z_{0}\right) \in B$ for all $t>T\left(z_{0}\right)$. Consequently, by Theorem 1.2 in Zhu and Smith (1994) 21] for the class of three-dimensional competitive systems, it has an orbitally asymptotically stable periodic solution.

The proof is complete.

\section{Numerical Simulation}

After providing all the analytical tools and qualitatively analysing the system for patterns on its dynamics, in this section, we will perform some numerical analysis on the model to verify the previous results.

4.1. Simulation tools. The numerical simulation is conducted on the programming language Julia through the package DifferentialEquation.jl, A Performant and Feature-Rich Ecosystem for Solving Differential Equations in Julia by Rackauckas and Nie (2017) [29.

In order to avoid any stiffness in the ODE models, the algorithm for the Method of Steps in Julia is set to Rosenbrock23, which is the same as the classic ODE solver ode23s in MATLAB.

The simulation is conducted on a system with a $2.0 \mathrm{GHz}$ dual core Intel core i5 with 16GB of RAM. 


\begin{tabular}{|c|c|c|}
\hline Parameters and Variables & & Values \\
\hline \multicolumn{3}{|l|}{ Dependent variables } \\
\hline$T$ & Uninfected CD4 ${ }^{+}$T-cell population size & $250 \mathrm{~mm}^{-3}$ \\
\hline$I$ & Infected CD4 ${ }^{+}$T-cell density & $50 \mathrm{~mm}^{-3}$ \\
\hline$V$ & Initial density of HIV RNA & $160 \mathrm{~mm}^{-3}$ \\
\hline \multicolumn{3}{|l|}{ Parameters and Constants } \\
\hline$s$ & Source term for uninfected CD $4^{+}$T-cells & 5 day $^{-1} \mathrm{~mm}^{-3}$ \\
\hline$d$ & Natural death rate of $\mathrm{CD} 4^{+}$T-cells & 0.01 day $^{-1}$ \\
\hline$a$ & Growth rate of $\mathrm{CD} 4^{+} \mathrm{T}$-cell population & 0.8 day $^{-1}$ \\
\hline$T_{\max }$ & Maximal population level of CD4 ${ }^{+} \mathrm{T}$-cells & $1500 \mathrm{~mm}^{-3}$ \\
\hline$\beta$ & Rate CD4 ${ }^{+}$T-cells became infected with virus & $2.4 \times 10^{-4} \mathrm{~mm}^{-3}$ \\
\hline$\alpha$ & Saturated mass-action term & 0.001 \\
\hline$\rho$ & Rate of cure & $0.01 \mathrm{day}^{-1}$ \\
\hline$\delta$ & Blanket death rate of infected $\mathrm{CD} 4^{+} \mathrm{T}$-cells & $0.3 \mathrm{day}^{-1}$ \\
\hline$q$ & Reproduction rate of the infected $\mathrm{CD} 4^{+} \mathrm{T}$-cells & $500 \mathrm{~mm}^{-3} \mathrm{day}^{-1}$ \\
\hline$c$ & Death rate of free virus & 8 day $^{-1}$ \\
\hline
\end{tabular}

TABLE 1. Preliminary values of variables and parameters for viral spread.

\begin{tabular}{lllll}
\hline Parameters & Original scenario & Scenario $\# 2$ & Scenario $\# 3$ & Scenario \#4 \\
$s$ & 5 & - & - & - \\
$d$ & 0.01 & - & - & - \\
$a$ & 0.8 & 8 & - & - \\
$T_{\max }$ & 1500 & - & - & - \\
$\beta$ & $2.4 \times 10^{-4}$ & - & 0.0024 & 0.0024 \\
$\alpha$ & 0.001 & 0.0001 & 0.000001 & 0.000001 \\
$\rho$ & 0.01 & 0.01 & - & - \\
$\delta$ & 0.3 & 5 & - & - \\
$q$ & 500 & - & 2.5 & 2.5 \\
$c$ & 8 & 1.3 & 3 & 1.3 \\
\hline
\end{tabular}

TABLE 2. Values of parameters for viral spread in different scenarios.

4.2. Simulation results. Within the range of parameters that are proven to be realistic in medical research, we investigate the behavior of the model within 4 different scenarios.

- The original scenario: In this scenario, the conditions 1,2 and 3 in Theorem 3.7 are satisfied. This means that, the positive equilibrium of the system 2.11 is globally asymptotically stable.

- Scenario \#2: In this scenario, the conditions 1, 2 and 4 in Theorem 3.7 are satisfied. This means that, the positive equilibrium of the system 2.1) is also globally asymptotically stable.

- Scenario \#3: In this scenario, the conditions 1 and 2 of Theorem 3.3 are satisfied. This means that, the positive equilibrium of the system 2.1] is locally asymptotically stable.

- Scenario \#4: In this scenario, the conditions 1 and 2 of Theorem 3.9 are satisfied. This means that, the positive equilibrium of the system (2.1) is orbitally asymptotically stable. 
Figure 1. The ODE model is locally asymptotically stable with parameters in the original scenario
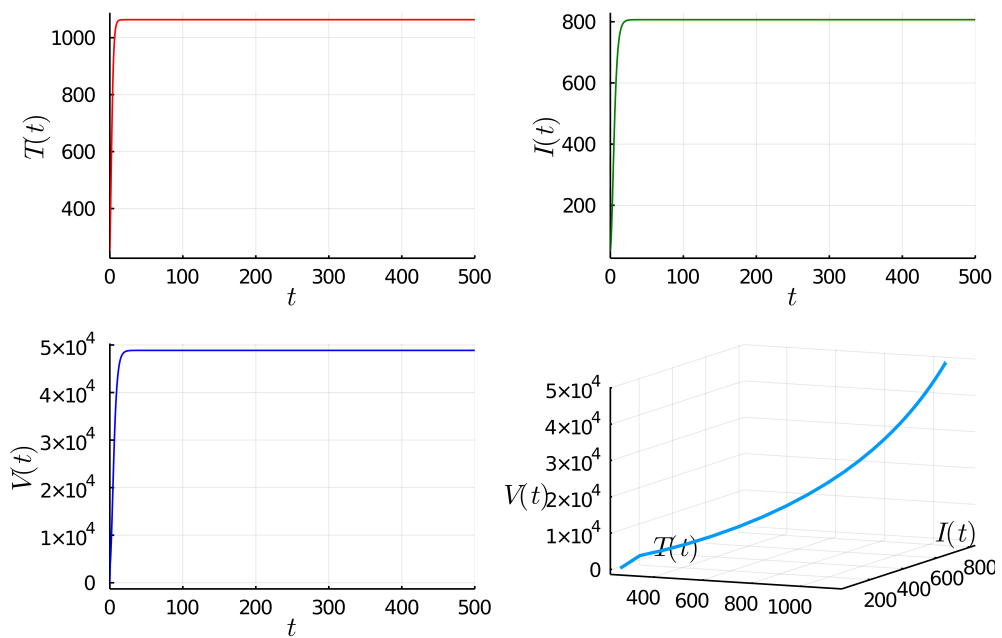

Figure 2. The ODE model is locally asymptotically stable with parameters in Scenario \#2
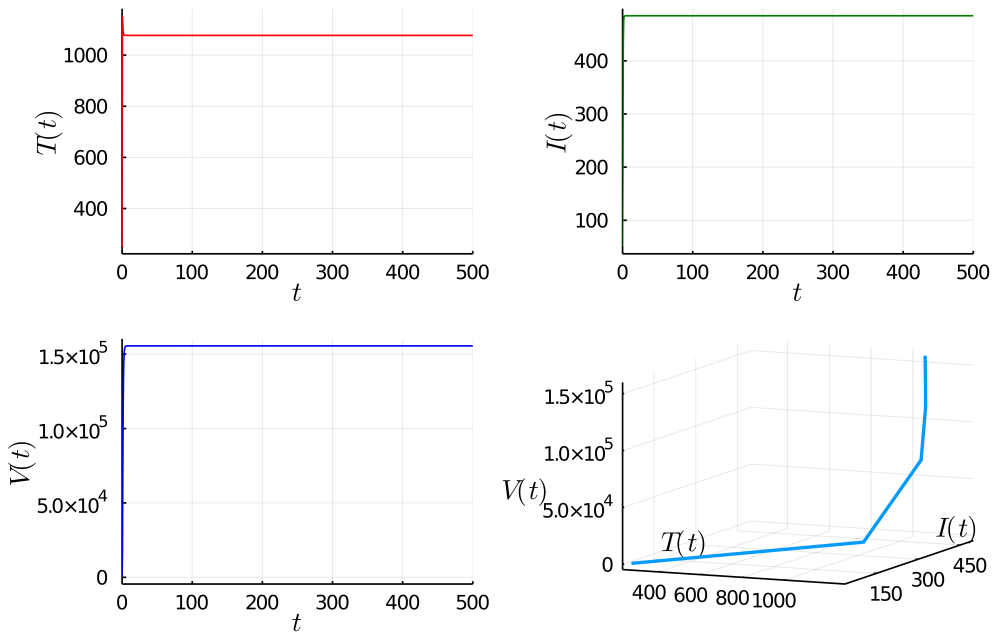

Acknowledgments. The authors would like to thank Tran Hai Yen NGUYEN, Molecular Biotechnology Laboratory, Faculty of Biology and Biotechnology, Ho Chi Minh University of Science, VNU - HCM, for providing valuable biological insights and ideas to support this research. The authors would also like to express their sincere gratitude towards the editor and reviewers for their valuable comments to improve the original manuscript. 
Figure 3 . The ODE model is locally asymptotically stable with parameters in Scenario \#3
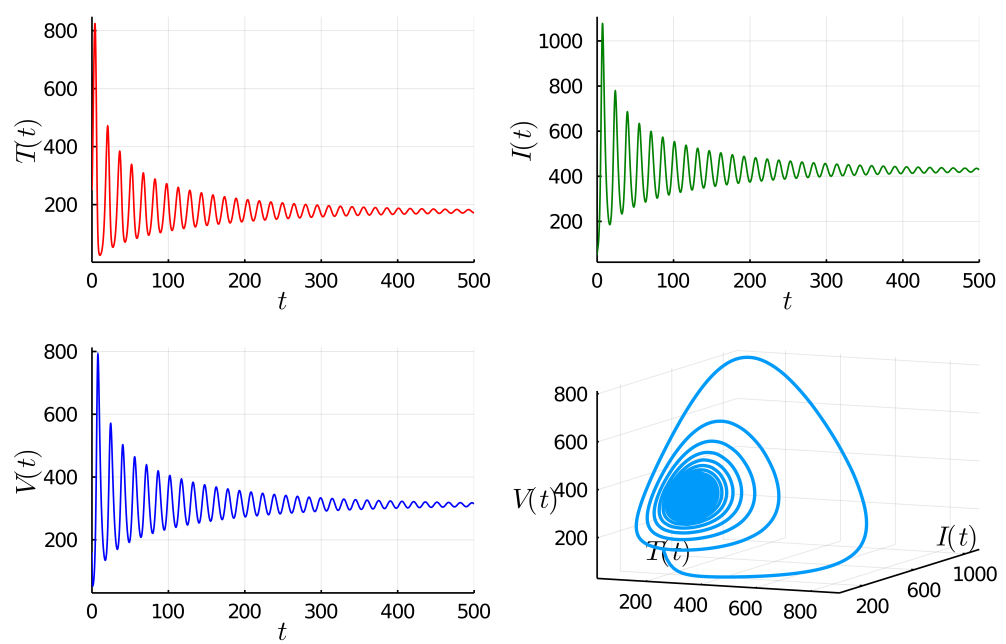

Figure 4. The ODE model is orbitally asymptotically stable with parameters in Scenario \#4
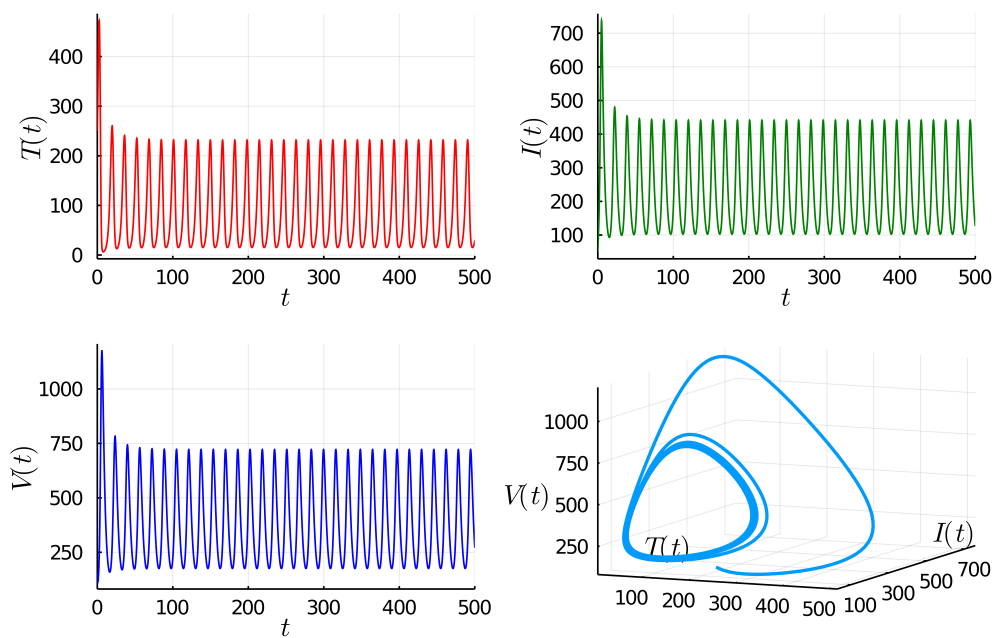

Appendix A. Detailed PRoof of USED theorems

Theorem A.1 (Gronwall, 1919). Let I denote an interval of the real line of the form $[a$, inf) or $[a, b]$ or $[a, b)$ with $a<b$. Let $\beta$ and $u$ be real-valued continuous functions defined on I. If $u$ is a differentiable function in the interior $I^{0}$ of I (the interval I without the end points a and possibly b) and satisfies the differential inequality

$$
u^{\prime}(t) \leq \beta(t) u(t), t \in I^{0}
$$


then $u$ is bounded by the solution of the corresponding differential equation $\nu^{\prime}(t)=$ $\beta(t) \nu(t)$ :

$$
u(t) \leq u(a) \exp \left(\int_{a}^{t} \beta(s) d s\right) .
$$

Theorem A.2 (Lyapunov's stability). Let a function $V(\mathbf{X})$ be continuously differentiable in a neighbourhood $U$ of the origin. The function $V(\mathbf{X})$ is called the Lyapunov function for an autonomous system

$$
\mathbf{X}^{\prime}=f(\mathbf{X})
$$

if the following conditions are met:

(1) $V(\mathbf{X})>0$ for all $\mathbf{X} \in U \backslash\{0\}$;

(2) $V(0)=0$;

(3) $\frac{d V}{d t} \leq 0$ for all $\mathbf{X} \in U$.

Then, if in a neighborhood $U$ of the zero solution $\mathbf{X}=0$ of an autonomous system there is a Lyapunov function $V(\mathbf{X})$ with a negative definite derivative $\frac{d V}{d t}$ for all $\mathbf{X} \in U \backslash\{0\}$, then the equilibrium point $\mathbf{X}=0$ of the system is asymptotically stable.

Theorem A.3 (Perron - Frobenius Theorem). 20, Let $A$ be an irreducible Metzler matrix (A Metzler matrix is a matrix whose all of its off-diagonal elements are non-negative). Then, $\lambda_{M}$, the eigenvalue of $A$ of largest real part is real, and the elements of its associated eigenvector $v_{M}$ are positive. Moreover, any eigenvector of $A$ with non-negative elements belongs the the span of $v_{M}$.

Theorem A.4 (Poincaré - Bendixson Theorem). 3

Given a differentiable real dynamical system defined on an open subset of the plane, every non-empty compact $\omega$-limit set of an orbit, which contains only finitely many fixed points, is either

- a fixed point,

- a periodic orbit, or

- a connected set composed of a finite number of fixed points together with homoclinic and heteroclinic orbits connecting these.

Moreover, there is at most one orbit connecting different fixed points in the same direction. However, there could be countably many homoclinic orbits connecting one fixed point.

Next, we will give the definition of an additive compound matrix and consider the particular case when it's a square matrix [1]. A survey of properties of additive compound matrices, along with their connections to differential equations have been investigated in [25, 26].

We will start with the definition of the $k$-th exterior power (or multiplicative compound) of an $n \times m$ matrix.

Definition A.1 (Multiplicative compound of a matrix). Let $A$ be an $n \times m$ matrix of real or complex numbers. Let $a_{i_{1}, i_{2}, \ldots, i_{k}, j_{1}, j_{2}, \ldots, j_{k}}$ be the minor of $A$ determined by the rows $\left(i_{1}, \ldots, i_{k}\right)$ and the columns $\left(j_{1}, \ldots, j_{k}\right), 1 \leq i_{1}<i_{2}<\ldots<i_{k} \leq n, 1 \leq$ $j_{1}<j_{2}<\ldots<j_{k} \leq m$. The $k$-th multiplicative compound matrix $A^{(k)}$ of $A$ is the $\left(\begin{array}{l}n \\ k\end{array}\right) \times\left(\begin{array}{c}m \\ k\end{array}\right)$ matrix whose entries, written in lexicographic order, are $a_{i_{1}, \ldots, i_{k}, j_{1}, \ldots, j_{k}}$.

In particular, when $A$ is an $n \times k$ matrix with columns $a_{1}, a_{2}, \ldots, a_{k}, A^{(k)}$ is the exterior product $a_{1} \vee a_{2} \vee \ldots \vee a_{k}$. 
In the case $m=n$, the additive compound matrices are defined as follows.

Definition A.2. Let $A$ be an $n \times n$ matrix. The $k$-th additive compound $A^{[k]}$ of $A$ is the $\left(\begin{array}{l}n \\ k\end{array}\right) \times\left(\begin{array}{l}n \\ k\end{array}\right)$ matrix given by

$$
A[k]=D(I+h A) \|_{h=0} .
$$

If $B=A^{[k]}$, the following formula for $b_{i, j}$ can be deduced from the equation A.4. For any integer $i=1, \ldots,\left(\begin{array}{l}n \\ k\end{array}\right)$, let $(i)=\left(i_{1}, i_{2}, \ldots, i_{k}\right)$ be the $i$-th member in the lexicographic ordering of all $k$-tuples of integers such that $1 \leq i_{1}<i_{2}<\ldots<i_{k} \leq n$. Then,

$$
b_{i, j}=\left\{\begin{aligned}
a_{i_{1}, i_{1}}+\ldots+a_{i_{k}, i_{k}} & \text { if }(i)=(j) \\
(-1)^{r+s} a_{i_{s}, j_{r}} \quad \text { if exactly one entry } i_{s} \text { in }(i) \text { does not occur in }(j) & \text { and } j_{r} \text { does not occur in }(i), \\
0 \quad & \text { if }(i) \text { differs from }(j) \text { in two or more entries. }
\end{aligned}\right.
$$

In the extreme cases when $k=1$ and $k=n$, we would have that $A^{[1]}=A$ and $A^{[n]}=\operatorname{tr}(A)$. For $n=3$, which is the case that we are considering in this paper, we would have the matrices $A^{[k]}, k=0,1,2$ as follows:

$$
A^{[1]}=A, \quad A^{[2]}=\left(\begin{array}{ccc}
a_{11}+a_{22} & a_{23} & -a_{13} \\
a_{32} & a_{11}+a_{33} & a_{12} \\
-a_{31} & a_{21} & a_{22}+a_{33}
\end{array}\right), \quad A^{[3]}=a_{11}+a_{22}+a_{33} .
$$

\section{REFERENCES}

[1] X. Song, and A. U. Neumann, Global stability and periodic solution of the viral dynamics, J. Math. Anal. Appl. 329 (2007) 281-297.

[2] R. V. Culshaw, and S. Ruan, A delay - differential equation model of HIV infection of CD4 ${ }^{+}$ T-cells, Math. Biosci. 165 (2000) 27-39.

[3] G. Teschl, Ordinary Differential Equations and Dynamical Systems (2012), American Mathematical Society, Providence, ISBN 978-0-8218-8328-0.

[4] R. J. De Boer, and A. S. Perelson, Target Cell Limited and Immune Control Models of HIV Infection: A Comparison 190 (1998) 201-214.

[5] A. S. Perelson, Modelling the interaction of the immune system with HIV, In: C. CastilloChavez (Eds), Mathematical and Statistical Approaches to AIDS Epidemiology (1989) 250, Springer, Berlin.

[6] A. S. Perelson, Dynamics of HIV Infection of CD4 ${ }^{+}$T-cells, Math. Biosci. 114 (1993) 81.

[7] A. S. Perelson, A. U. Neumann, M. Markowitz, J. M. Leonard, and D.D. Ho, HIV-1 dynamics in vivo: virion clearance rate, infected cell life-span, and viral generation time, Science 271 (1996) 1582.

[8] A. S. Perelson, and P. W. Nelson, Mathematical analysis of HIV-1 dynamics in vivo, SIAM Rev. 41 (1999) 3.

[9] J. E. Mittler, B. Sulzer, A. U. Neumann, and A.S. Perelson, Influence of delayed viral production on viral dynamics in HIV-1 infected patients, Math. Biosci. 251 (1998) 143.

[10] J. E. Mittler, M. Markowitz, D. D. Ho, and A. S. Perelson, Improved estimates for HIV-1 clearance rate and intracellular delay, AIDS 13 (1999) 1415.

[11] R. M. Anderson, and R. M. May, Complex dynamical behavior in the interaction between HIV and the immune system, In: A. Goldbeter (Eds), Cell to Cell Signalling: From Experiments to Theoretical Models (1989) 335, Academic Press, New York. 
[12] A. R. McLean, and T. B. L. Kirkwood, A model of human immunodeficiency virus infection in T-helper cell clones, J. Theoret. Biol. 147 (1990) 177.

[13] M. A. Nowak, and R. M. Bangham, Population dynamics of immune responses to persistent viruses, Science 272 (1996) 74.

[14] D. E. Kirschner, Using mathematics to understand HIV immune dynamics, Notices Amer. Math. Soc. 43 (1996) 191.

[15] Y. Kuang, Delay-Differential Equations with Applications in Population Dynamics (1993), Academic Press, New York.

[16] A. V. M. Herz, S. Bonhoeffer, R. M. Anderson, R. M. May, and M. A. Nowak, Viral dynamics in vivo: limitations on estimates of intracellular delay and virus decay, Proc. Natl. Acad. Sci. USA 93 (1996) 7247.

[17] J. Tam, Delay effect in a model for virus replication, Math. Med. Biol. 16 (1999) 29.

[18] T. B. Kepler, and A.S. Perelson, Cyclic re-entry of germinal center B cells and the efficiency of affinity maturation, Immunology Today, 14 (1993) 412-415.

[19] J. K. Percus, O. E. Percus, and A. S. Perelson, Predicting the size of the T -cell receptor and antibody combining region from consideration of efficient self-nonself discrimination, Proc. Natl. Acad. Sci. USA 90 (1993) 2691-2695.

[20] F. R. Gantmacher, The Theory of Matrices (1959), Chelsea Publishing Company, New York.

[21] H. R. Zhu, H. L. Smith, and M. W. Hirsch, Stable periodic orbits for a class of three dimensional competitive systems, J. Differential Equations 110 (1994) 143-156.

[22] M. W. Hirsch, System of differential equations which are competitive or cooperative, IV, SIAM J. Math. Anal. 21 (1990) 1225-1234.

[23] G. Butler, H. I. Freedman, and P. Waltma, Uniform persistence system, Proc. Amer. Math. Soc. 96 (1986) 425-430.

[24] H. L. Smith, and H. Thieme, Convergence for strongly ordered preserving semiflows, SIAM J. Math. Anal. 22 (1991) 1081-1101.

[25] J. S. Muldowney, Compound matrices and ordinary differential equations, Rocky Mountain J. Math. 20 (1990) 857-872.

[26] Y. Li, and J. S. Muldowney, Global stability for the SEIR model in epidemiology, Math. Biosci. 125 (1995) 115-164.

[27] International Committee on Taxonomy of Viruses, Taxonomy (2019), National Institutes of Health, URL: https://talk.ictvonline.org/taxonomy/

[28] R. Harvey, Microbiology (3rd ed.) (2012) 295-306, Lippincott Williams \& Wilkins, Philadelphia, PA.

[29] C. Rackauckas, and Q. Nie, DifferentialEquations.jl - A Performant and Feature-Rich Ecosystem for Solving Differential Equations in Julia, J. Open Res. Softw. 5(1) 201715.

Hoang Anh NGO,

École Polytechnique, Institut Polytechnique de Paris, 91120 Palaiseau, France, Phone: (+33) 7618680 94, Orcid ID: 0000-0002-7583-753X

Email address: hoang-anh.ngo@polytechnique.edu

Hung DANg NGUYEN,

Vietnamese - German Faculty of Medicine (VFGM), Pham Ngoc Thach University of Medicine, Ho Chi Minh City 710000, Vietnam, Phone: (+84) 8392678 24, Orcid ID: 0000-0002-3169-6499

Email address: 1851010243@pnt.edu.vn

Mehmet DiK,

Department of Mathematics and Computer Sciences, Beloit College, Wi 53511, United

States, Phone: (+1) 608-363-2566, Orcid ID: 0000-0003-0643-2771

Email address: dikm@beloit.edu 